\title{
INVESTIGACIONES
}

\section{Comenzar y permanecer \\ Narrativas de dos docentes de escuelas con alto índice de vulnerabilidad*}

\author{
Beginning and staying \\ Narrative from two teachers from schools with high vulnerability indexes
}

\author{
Alessandra Díaz Sacco ${ }^{a}$ \\ ${ }^{a}$ Facultad de Educación, Pontificia Universidad Católica de Chile. andiaz@uc.cl
}

\begin{abstract}
RESUMEN
Más del $40 \%$ de los docentes chilenos abandona la profesión durante los primeros años de ejercicio profesional. Adicionalmente, al considerar entornos de mayor vulnerabilidad escolar esta problemática se ve agudizada. Considerando este contexto, este estudio propone un acercamiento a las experiencias de permanencia de dos profesores principiantes que se desempeñan en escuelas chilenas con altos índices de vulnerabilidad. A partir de un enfoque ecológico de la agencia docente fue posible adentrarse en la comprensión de dichas experiencias de forma multidimensional e integrada. Se utiliza una aproximación biográfica narrativa que permite poner en el centro del análisis la experiencia de permanencia. En base a las narraciones de Andrea e Ignacio se evidencia un entramado continuo de decisiones que los llevan a permanecer en sus escuelas, que no está exento de momentos de discontinuidad y cuestionamientos.
\end{abstract}

Palabras claves: movilidad docente, docentes principiantes, carrera docente.

\begin{abstract}
More than $40 \%$ of Chilean teachers leave the profession during the first years of their professional practice. Also, when considering environments of greater vulnerability in schools, this problem is exacerbated. In this context, this study proposes an approach to the permanence experiences of two beginning teachers who work in Chilean schools with high vulnerability rates. Based on an ecological approach of the teacher agency it was possible to enter into an understanding of such experiences in a multidimensional and integrated manner. The study uses a biographical narrative approach that puts the experience of permanence at the center of the analysis. Based on the narratives of Andrea and Ignacio, a continuous network of decisions is evidence that leads them to remain in their schools, which is not exempt from moments of discontinuity and questioning.
\end{abstract}

Key words: teacher mobility, beginning teachers, teacher career.

\footnotetext{
* Este estudio cuenta con el financiamiento de la Agencia Nacional de Investigación y Desarrollo (ANID). Programa de
} Becas. Doctorado Nacional 2017-21171112. 


\section{INTRODUCCIÓN}

El estudio de las decisiones de movilidad de profesores ha tomado relieve a nivel internacional y nacional, en especial al considerar las altas y tempranas tasas de abandono de la profesión docente en algunas naciones, realzando la necesidad de avanzar de manera urgente hacia sistemas educativos que logren la retención y desarrollo de sus maestros en el largo plazo (Ávalos y Valenzuela, 2016; OCDE, 2005). En este contexto, es interesante analizar el caso chileno, dado que el país alcanza una de las tasas más altas de abandono a nivel global, superiores al $40 \%$ durante los primeros años de ejercicio de la profesión docente (Ávalos et al., 2016; Valenzuela y Sevilla, 2013).

Adicionalmente, se ha determinado que la decisión de abandonar la enseñanza acontece como un proceso gradual, en el cual el primer año de ejercicio de la profesión es clave para la continuidad de los profesores en el sistema educativo (Hobson et al., 2007). En este ámbito se ha definido el inicio de la docencia como un período particularmente crítico, ya que aproximadamente un $20 \%$ de los profesores chilenos se retira del sistema educativo durante el primer año de ejercicio de la profesión (Valenzuela et al., 2013). De modo complementario, un estudio reciente en torno a la rotación de profesores novatos en el país señala que el $34,5 \%$ de los docentes principiantes ya no trabajará en el mismo establecimiento educacional luego de un año, cifra que aumenta al 50,5\% luego de tres años (Carrasco, Godoy y Rivera, 2017).

También, es clave puntualizar que, en contextos de mayor vulnerabilidad, en los cuales los docentes trabajan con un porcentaje alto de estudiantes con desventajas socioeconómicas, el abandono y rotación de maestros principiantes es especialmente crítico (Borman y Dowling, 2008). Se ha señalado que aquellos docentes noveles que se desempeñan en entornos desafiantes (Kelly y Northrop, 2015), en escuelas con bajo rendimiento académico, en contextos vulnerables o en entornos económicamente desfavorecidos (Feng, 2010) tienen mayores probabilidades de rotar o abandonar dichas escuelas. En particular, para el caso chileno se ha propuesto que los profesores de enseñanza media y aquellos que trabajan en establecimientos con un índice de vulnerabilidad escolar (IVE-SINAE ${ }^{1}$ ) del 80 al 100\% presentan mayor riesgo de abandonar tempranamente. De esta forma, los profesores que inician su carrera profesional en escuelas con alto índice de vulnerabilidad, en promedio dejan su primer trabajo después de 2,2 años de ejercicio docente, significativamente antes que el resto de sus colegas que lo harán luego de 3 años (Carrasco et al., 2017).

En este escenario de significativo abandono y rotación docente a nivel nacional, durante el año 2016 se promulga la Ley de Desarrollo Profesional Docente en Chile, la cual considera la incorporación de un aumento de los requisitos de ingreso a las carreras de pedagogía y la exigencia de la acreditación de las instituciones formadoras, elementos que junto a otros que propone esta Ley buscan asegurar el ingreso y permanencia de postulantes idóneos a la profesión (CPEIP, 2017). Esto es consistente con la evidencia internacional de países referentes en materia educacional, en los cuales la selectividad de los programas de formación es altamente valorada como elemento que ha permitido mejorar las capacidades docentes (Mourshed, Chijioke y Barber, 2010) y cuya exigencia

Índice de Vulnerabilidad Escolar - Sistema Nacional de Asignación con Equidad. El índice es calculado considerando un conjunto de criterios que permite identificar la condición de vulnerabilidad de la población estudiantil de una institución escolar, en base a diferentes fuentes de información de cada estudiante. 
atrae a los mejores candidatos (Barber y Mourshed, 2008). Sin embargo, el aumento de los requisitos de ingreso y los mayores requerimientos en la formación de estos futuros profesionales podrían llegar a limitar la cantidad de nuevos maestros disponibles para el recambio de aquellos docentes que dejan la enseñanza (Ávalos et al., 2016) provocando mayores presiones al sistema educativo, las cuales podría llegar a ser más agudas en sectores de mayor pobreza y vulnerabilidad (Boyd, Lankford, Loeb y Wyckoff, 2005).

En respuesta a la problemática de abandono y rotación, un importante y creciente número de indagaciones se ha dedicado a esclarecer cuáles serían los factores que determinan de forma más precisa las decisiones de abandono, rotación o permanencia de los profesores, contando hoy en día con un cuerpo importante de investigaciones que han definido factores individuales y contextuales que se encontrarían relacionados a la movilidad docente (Bettini et al., 2017; Dupriez, Delvaux y Lothaire, 2016; Gaikhorst, Beishuizen, Roosenboom y Volman, 2017; Harfitt, 2015; Heikonen, Pietarinen, Pyhältö, Toom y Soini, 2016; Kelly et al., 2015; Lavigne, 2014; entre otros). Sin embargo, autores como Schaefer, Long y Clandinin (2012) y Day (2008) advierten que es necesario transitar desde la comprensión de factores hacia una mirada más integrativa, que permita proponer condiciones sistémicas para el desarrollo y permanencia de los docentes en el sistema educativo. Asimismo, se ha propuesto que, sería importante transitar hacia una comprensión que se centre en las experiencias de los maestros más que en factores individuales o contextuales relacionados a las decisiones de los docentes frente a su trayectoria profesional (Clandinin, Schaefer y Downey, 2014).

Considerando los antecedentes expuestos, este trabajo se propuso comprender las decisiones de permanencia de dos docentes principiantes que se desempeñan en contextos de mayor adversidad, utilizando un marco teórico que admita una aproximación integrada y profunda de la experiencia vivida. Por lo tanto, aproximarse a la comprensión del fenómeno de la permanencia en aquellos docentes que presumiblemente se encuentran en mayor riesgo de abandono pero que han permanecido en sus puestos de trabajo. Concretamente, adentrarse en una comprensión ecológica de las decisiones de permanencia de profesores principiantes chilenos que se desempeñan en escuelas de alto IVE-SINAE, guiado por la pregunta: ¿Cuáles son las narrativas de permanencia en el sistema escolar chileno de profesores principiantes en escuelas con alto índice de vulnerabilidad escolar, atendiendo a las relaciones entre las experiencias personales y profesionales, las aspiraciones con respecto al quehacer docente y el despliegue cotidiano de la labor profesional de los maestros?

\section{UNA APROXIMACIÓN ECOLÓGICA A LAS DECISIONES DE PERMANENCIA}

En este trabajo se opta por una aproximación a las decisiones de permanencia utilizando un enfoque ecológico de la agencia docente (Priestley, Biesta y Robinson, 2015). Este enfoque permite un abordaje del fenómeno considerando la integración de características personales de los docentes con sus entornos de despliegue, y de este modo analizar la permanencia en las escuelas considerando una variedad de dimensiones y sus relaciones. Esta aproximación considera una mirada de la agencia como un fenómeno emergente, que involucra las capacidades, habilidades, saberes, creencias, entre otros, del individuo en interacción con las condiciones contextuales del quehacer docente. Por lo tanto, la agencia es vista como un fenómeno dinámico y único (Priestley et al., 2015). 
Esta investigación adoptó esta aproximación teórica con la finalidad de analizar de forma integrada y en profundidad las decisiones de permanencia de docentes principiantes, integrando las tres dimensiones propuestas por el enfoque ecológico de la agencia docente, estás son: dimensión iterativa, que recoge las experiencias e historias personales y profesionales de los docentes; dimensión evaluativa práctica, que considera las condiciones de despliegue cotidiano de los docentes; y dimensión proyectiva, que atiende a las aspiraciones de corto y largo plazo de los maestros. En este enfoque de agencia, esta no se entiende como algo que es poseído por los individuos, sino que es alcanzado a partir de la interacción activa de los sujetos con aspectos de su contexto, y en este sentido, se concibe como una comprensión ecológica de la agencia. Por lo tanto, la forma de comprender la agencia se centra en la interacción dinámica de las tres dimensiones antes propuestas, que tiene en cuenta cómo esta interacción varía en diferentes contextos de despliegue profesional (Biesta y Tedder, 2007).

\section{METODOLOGÍA}

Se propone una aproximación cualitativa, de tipo inductiva y exploratoria, en específico siguiendo un enfoque biográfico narrativo adentrarse en las experiencias pasadas, presentes y en las proyecciones de dos docentes principiantes que se desempeñan como maestros en escuelas con un índice de vulnerabilidad escolar mayor al 80\%. Por lo tanto, el objetivo que se plantea es indagar en las continuidades y discontinuidades de las narrativas de permanencia de profesores principiantes en escuelas con alto índice de vulnerabilidad.

Se selecciona para esto una aproximación biográfica narrativa ya que permite explorar en las experiencias vividas y las perspectivas que los individuos tienen de sus vidas, considerando sus historias pasadas, su presente y la proyección futura, poniendo atención en cómo se configura el significado que los sujetos dan a las historias que narran (Denzin, 1989). En este sentido, un enfoque biográfico narrativo permite indagar en las dimensiones propuestas por el enfoque ecológico de agencia docente, atendiendo a las condiciones estructurales y contextuales del quehacer docente y a las experiencias personales de los maestros.

\subsection{MUESTRA}

La selección de la muestra se realizó a partir de un muestreo no probabilístico, en cadenas o por redes, el cual permitió la identificación de maestros que cumplían con las características esperadas para el estudio (Hernández, Fernández y Baptista, 2014). La determinación de los criterios de inclusión de los profesores elegibles se basa en las características propuestas por el reciente estudio de Carrasco, Manzi y Treviño (2018), en el cual se caracteriza a los docentes principiantes que presentan mayor riesgo de abandonar su primer empleo en las escuelas chilenas. Se consideran las características desarrolladas en dicha investigación con la finalidad de contar con relatos de docentes que, aun teniendo los atributos esperados para abandonar prematuramente, permanecen en sus puestos de trabajo, distinguiendo que en ellos la decisión de permanencia podría presumirse se encuentra en mayor riesgo y requiere de una actualización recurrente.

Por lo tanto, los candidatos para este estudio debieron cumplir al menos con los siguientes criterios de inclusión: docentes de educación secundaria en escuelas de la 
Región Metropolitana con un rango IVE-SINAE que se encuentre entre el 80 al 100\%. Se considera relevante el nivel de enseñanza y el índice de vulnerabilidad de la escuela, dado que destacan dentro de los factores más críticos en la probabilidad de retiro temprano de los docentes (Valenzuela et al., 2013; Carrasco et al., 2018). Adicionalmente, para resguardar el trabajo con docentes noveles, los maestros participantes de este estudio se encuentran desempeñando la docencia hasta un máximo de su quinto año de ejercicio. En el estudio participaron dos profesores principiantes, los cuales se caracterizan en la Tabla 1.

Tabla 1. Caracterización de la muestra

\begin{tabular}{|c|c|c|c|c|c|}
\hline Profesor/a & Edad & $\begin{array}{c}\text { Años totales } \\
\text { de docencia }\end{array}$ & $\begin{array}{c}\text { Años de permanencia } \\
\text { en el establecimiento } \\
\text { actual }\end{array}$ & $\begin{array}{c}\text { IVE -SINAE del } \\
\text { establecimiento }\end{array}$ & $\begin{array}{c}\text { Asignatura que } \\
\text { imparte }\end{array}$ \\
\hline Andrea & 27 & 5 & 5 & $88 \%$ & $\begin{array}{c}\text { Lenguaje y } \\
\text { Comunicación }\end{array}$ \\
\hline Ignacio & 32 & 5 & 5 & $91 \%$ & Artes Visuales \\
\hline
\end{tabular}

Fuente. Elaboración propia.

\subsection{DATOS}

La producción de datos consideró la realización de 6 entrevistas narrativas a cada uno de los docentes pertenecientes a la muestra, las cuales fueron efectuadas por la investigadora responsable del estudio con una frecuencia de una entrevista mensual, teniendo una duración aproximada de entre 60 a 90 minutos cada una de ellas. Cabe mencionar que el diseño fue iterativo, dado que las entrevistas consideraban los hallazgos principales de la entrevista anterior. Mishler (1986) propone que este tipo de entrevistas son una forma en la cual los entrevistados pueden desarrollar relatos de vida ${ }^{2}$, lo cual permite generar informes detallados en lugar de respuestas breves o generales.

En concreto, para cada una de las entrevistas se consideró la construcción de una pauta semiestructurada que fue la guía de la investigadora responsable. Para esto se siguieron los planteamientos de Riessman (2008) en relación con la construcción de entrevistas narrativas, quien propone generar preguntas amplias que abran temas y permitan a los profesores entregar respuestas de la manera que les parezca significativa. Las pautas fueron sometidas a un proceso de validación del cual participaron siete expertos, en resguardo de la validez de los instrumentos construidos. Adicionalmente, se realizó un pilotaje de las pautas con un profesor principiante distinto a los de la muestra pero que cumplía con las mismas características de esta.

Las pautas de entrevistas en su conjunto consideraron la indagación en las distintas dimensiones propuestas por el enfoque ecológico de la agencia docente. Por lo tanto, se

"El relato de vida corresponde a la enunciación -escrita u oral- por parte de un narrador de su vida o parte de ella" (Cornejo, Mendoza y Rojas, 2008). 
consideró examinar distintas áreas temáticas contenidas en cada una de las dimensiones propuestas por el enfoque, las cuales se detallan en la Tabla 2.

Tabla 2. Dimensiones propuestas por el enfoque ecológico de la agencia docente

\begin{tabular}{|l|l|l|}
\hline Dimensión iterativa & Dimensión evaluativa práctica & Dimensión proyectiva \\
\hline - Historias de vida de los & Cultural & Aspiraciones y \\
profesores. & - Ideas, valores, creencias, & proyecciones a corto y \\
- Experiencias & discursos. & \\
profesionales. & Estructural & \\
& - Relaciones, roles, poder, \\
& estructuras relacionales. & \\
& Material & \\
& - Recursos, entorno. & \\
\hline
\end{tabular}

Fuente. Priestley, Biesta y Robinson (2015).

Adicionalmente, se complementaron las entrevistas narrativas con la técnica de photo elicitation (Harper, 2002), la cual consiste en presentar fotografías a los profesores que permitan evocar elementos más profundos de la conciencia de los docentes. Esta técnica permitió acceder a una mayor profundidad analítica en las diferentes temáticas presentadas a los docentes.

Finalmente, el análisis de los datos se llevó a cabo utilizando un método temático (Riessman, 2008), el cual permitió reconstruir narrativamente las experiencias de permanencia. En este sentido, la unidad de análisis considerada fue el narrador en cuanto a su experiencia de permanencia. Considerando lo anterior, se procedió del siguiente modo: en un primer momento se trabajó con cada una de las entrevistas de forma separada, ordenando los episodios relevantes en un relato biográfico cronológico. Posteriormente, una vez que el proceso fue completado para cada una de las entrevistas, se procedió a la identificación de temáticas subyacentes y se codificaron. Esto permitió la construcción de biografías docentes, que resaltan e interrelacionan dimensiones particulares incumbidas en las experiencias de permanencia en las escuelas. Finalmente, se presentaron las biografías construidas a los profesores, lo que permitió una negociación de los temas y los significados entre el narrador y la investigadora, este procedimiento permitió la validación de las historias construidas a través de sus propios protagonistas.

\section{RESULTADOS}

Los resultados se presentan privilegiando un despliegue de la experiencia de permanencia de cada uno de los profesores de la muestra. Posteriormente, en la sección de discusión se presentan continuidades y discontinuidades en la experiencia de permanencia, con la finalidad de caracterizar algunos ejes temáticos relevantes que iluminen la comprensión del fenómeno de la permanencia. 


\subsection{LA HISTORIA DE ANDREA}

Narrar la historia de Andrea en la pedagogía y las decisiones que ha ido tomando requiere volver hacia atrás, a su infancia, que se encuentra marcada por la relación con su madre y abuela materna. Ellas, cada una en un rol determinado, asumen el cuidado de Andrea desde su nacimiento hasta los 10 años:

Mi mamá, como madre soltera, tuvo que salir a trabajar para llevar la comida a mi casa y mi abuelita me crió, siendo que le faltaba un brazo y que tenía vista sólo de un ojo.

A los 10 años, la abuela de Andrea fallece, provocando una de las primeras crisis en su vida. Comienza así una etapa de soledad y resiliencia, que ella destaca como formadora de su personalidad y definitoria al momento de apoyar a niños y jóvenes de contextos vulnerables:

Cuando mi abuelita murió tuvimos que salir adelante las dos (ella y su madre). Entonces cuando hago clases siempre pienso en eso; en el modelo de esfuerzo y de darlo todo por lo que amas, por la pasión que tienes.

Posteriormente, durante su adolescencia, Andrea, que tenía muy buen rendimiento escolar y pasión por la lectura, tomó la primera decisión que la acercaría al mundo de la docencia del Lenguaje: seguir el electivo humanista en su colegio, en el cual además conoció a sus primeras influencias sobre la carrera de pedagogía, su profesora de electivo y su profesor de historia:

Siempre han sido mi modelo de profesores y cuando yo empiezo a estudiar la pedagogía es como... 'quiero ser como ellos'.

Andrea ingresa a la Universidad a estudiar Licenciatura en Letras Hispánicas, y aunque su intención inicial fue solamente adquirir conocimientos sobre literatura y lingüística, en uno de sus cursos universitarios comienza a cuestionarse el rol de la lectura en la formación del ser humano y cómo ese acto (para ella) tan individual, podía conectarse con la realidad a nivel social. Esto la moviliza y termina de convencer de proseguir estudios de pedagogía. Aunque esta decisión no estuvo exenta de dudas sobre si misma y sus capacidades:

Yo pensaba ‘¿me la podré o no me la podré?’. Pero haciendo clases en el Preuniversitario, durante mi época universitaria, me di cuenta de que esto es lo mío, me apasiona y que tengo que seguir, aunque sea difícil.

Al finalizar la formación docente, Andrea postuló a diversas instituciones educacionales en busca de su primer trabajo, pero todas compartían una característica: atendían las necesidades de niños y comunidades de contextos vulnerables. Ella tomó esta determinación luego de realizar su práctica profesional en un colegio particular pagado en una comuna acomodada de Santiago, donde el tipo de estudiantes y apoderados no calzaron con las expectativas y la realidad que buscaba Andrea:

Quizás es algo negativo de mí, porque yo nunca me he atrevido a salir de mi zona de Confort y no me atrevo a cambiarla porque tengo muchos prejuicios en torno a los 
colegios que tienen mucho dinero y que son particulares pagados. Pero cuando empecé a hacer clases dije: Si yo quiero hacer algo por Chile, tengo que partir por los colegios que más me necesitan, entonces voy a buscar a las corporaciones que trabajen con los chiquillos más problemáticos del país acá en Santiago.

En diciembre del año 2014, un colegio particular subvencionado ubicado en una zona céntrica de Santiago y con uno de los índices de vulnerabilidad más altos de la comuna, le abrió las puertas y la integró como profesora de Lenguaje y Comunicación.

Cuando postulé me gustó mucho la corporación y me dije 'ya, ahora tengo que tirarme a los leones no más'.

En el establecimiento, ella se hace cargo de la asignatura de Lenguaje y Comunicación para todos los cursos de enseñanza media, también asumió la jefatura de un tercero medio y el rol de profesora asesora del centro de alumnos. Un elemento que la sorprende gratamente al poco tiempo de ingresar a la institución es que es un espacio que ella denomina multicultural.

Cuando yo llego a este colegio se me abre una perspectiva nueva, algo que yo no había pensado antes, sentí que era más latinoamericana que chilena, eso me lo entregó este colegio. Aquí la mayoría de los niños no son chilenos. Vi con claridad que los inmigrantes lo pasan pésimo, y yo lo sé porque veo a mis alumnos, conozco a sus familias, y yo creo que es un tema muy importante, del cual debemos hacernos cargo. Este colegio es un espacio que acoge esa realidad, me da la posibilidad de trabajar con chiquillos de otras culturas, eso es algo muy importante para mí y es una razón que no imaginé, pero que me mantiene aquí.

Sin embargo, el primer año laboral de Andrea no estuvo exento de problemas. La adaptación a la institución, al contexto y dinámica de los alumnos, las exigencias de la corporación y sus nuevas responsabilidades requirieron de mucha dedicación para poder avanzar. Recuerda, por ejemplo, que:

Cuando yo llegue me costó adaptarme, porque antes que yo llegara, había una profesora que era muy querida por los estudiantes. Entonces, cuando yo entro al colegio y me enfrento a toda la enseñanza media, me costó mucho entrar con ellos a nivel de relación.

No obstante, la personalidad de Andrea y su perseverancia, la hicieron sortear estos primeros obstáculos sin mayores problemas, convirtiéndose en una profesora respetada por su alumnado y colegas:

Tuve mucho apoyo de parte de mi coordinadora y directora de ese entonces, de mis colegas también y de a poco nos fuimos acostumbrando todos. Ahora el ambiente es muy bueno.

Hoy en día, una de las características del colegio que Andrea valora por sobre muchas otras y que fue ganando con el tiempo es la posibilidad de crear y administrar de forma autónoma sus clases y su planificación. 
En este colegio, como yo soy el departamento de lenguaje en media, todas las decisiones pasan por mí. Hay muchas cosas que ya no hago y que tuve que hacer en un principio, como seguir la decisión a nivel del plan lector o de pruebas. Hoy yo hago mis propias pruebas.

Otro desafío importante para Andrea en su primer año fue adaptarse al contexto en el cual se desenvuelven los alumnos del colegio. El establecimiento se encuentra cercano a lugares conocidos como foco de tráfico de drogas, alcohol y violencia. Estas fueron temáticas con las que Andrea tuvo que comenzar a trabajar desde su entrada al colegio, puesto que por la edad de los alumnos con los que trabaja, su grado de vulnerabilidad a este ambiente es mayor:

Cuando yo llegué acá, las drogas, eran un tema. Los estudiantes tenían muy naturalizado llegar bajo los efectos de una pastilla o de la marihuana, se juntaban afuera, ahí hacían todo lo que querían y acá uno los recibía en esas condiciones. Con los años ha ido mejorando por lo menos acá, dentro del colegio.

Aunque ella menciona que el tema actualmente ha mejorado, reconoce la vulnerabilidad de sus alumnos al respecto y es enfática en su vocación de "salvarlos" y sacarlos adelante desde la docencia y el apoyo psicológico y emocional. Razón que ella menciona como un elemento clave para mantenerse en el establecimiento por tanto tiempo:

Cuando ellos se dan cuenta que ya son alguien en la vida, que tienen buenas características, que tienen potencial, que pueden hacer lo que ellos quieran, eso para mí es salvarlos, es decir, que se den cuenta que tienen algo bueno adentro suyo, que no tienen que llegar a ser alguien profesionalmente hablando para ser alguien en la vida, que ya lo son en este momento.

La carrera docente de Andrea en el colegio tuvo un quiebre el año 2017, que casi la lleva a abandonar su trabajo y cambiar rotundamente de establecimiento. Ese año los puestos del director y coordinadora académica del establecimiento fueron ocupados por dos personas con quienes tuvo una muy mala relación laboral. El quiebre se produjo durante un período de paro de los alumnos del colegio, en el cual Andrea, como profesora acompañante del centro de alumnos, tuvo un papel protagónico y empático para con los jóvenes. Esto no fue tomado de buena forma por las autoridades del establecimiento, quienes cambiaron la forma en que la trataban y desencadenaron en ella una serie de trastornos ansiosos que la llevaron a pensar en renunciar:

El trato hacia mí era violento, ya rayaba en la violencia y yo dije no, no puedo seguir trabajando aquí. Fue terrible para mí. Lo pasé muy mal, con cuadros de ansiedad, ataques de pánico en mi casa, muy mal. Somaticé absolutamente todo el estrés que tenía y dije, 'no puedo, a pesar de que amo este colegio, amo a mis niños, a mis estudiantes, me llevo muy bien con mis colegas, yo no puedo seguir acá'.

Cuando ya había comenzado el proceso de selección en otro establecimiento, Andrea recibe la noticia de que el director del colegio había sido desvinculado de su cargo y que 
con él se iría también la coordinadora académica del establecimiento. Con el recambio del equipo de gestión decide permanecer y pudo volver a contar con la autonomía que buscaba y retomar la rutina, cómoda y tranquila, que aplica hasta la actualidad:

En este momento, me gusta que tengo libertad de trabajo en un cierto límite y que las reglas son claras. Con esta directora yo sé lo que tengo que hacer y sé lo que no tengo que hacer y eso para mí también implica claridad de roles.

No obstante, estas problemáticas, Andrea volvió a disfrutar cada vez más el contexto en el que se desenvuelve profesionalmente y renueva su compromiso:

Si me gusta estar aquí es por los estudiantes, es eso lo que más me mueve.

Día a día, Andrea intenta desarrollar en sus alumnos los valores que para ella han sido fundamentales para avanzar en la vida y más allá de los contenidos propios del curriculum y de sus clases de Lenguaje, ella intenta siempre que sus alumnos se lleven de su rol como docente una guía en temáticas más allá del aula de clases:

Si me he quedado aquí todo este tiempo es por el tipo de relaciones que puedo establecer con los estudiantes, en el sentido de la confianza. Que son respetuosos. Que digan que somos flojos, que tenemos malas notas, pero respetuosos siempre, que nunca le hemos faltado el respeto a nadie. Y son esforzados a pesar de todo, son chiquillos que lo pasan mal en su día a día y al final encuentro sentido a mi trabajo aquí. Por eso ese año en que yo estuve a punto de renunciar, me quedé por ellos.

Es por una buena relación con su alumnado y por la posibilidad de realizarse profesionalmente desde un punto de vista cómodo y "a su manera" que Andrea comenta feliz que ha permanecido en el colegio por tanto tiempo. Pero sobre todas las cosas, una de las mayores motivaciones para superar todos los obstáculos que se le puedan presentar en el camino, es el cariño e identificación que tiene por el contexto en el que se desenvuelven sus alumnos:

Yo vengo de una familia donde la mamá es proveedora, madre soltera, jefa de hogar, papá desaparecido, criada con una abuela hasta los 10 años, después yo sola, llegar a la casa sola, estudiar sola, con una mamá siempre muy preocupada, entonces, tengo estudiantes que son muy semejantes a mí en ese sentido, y eso me moviliza.

Actualmente, si a Andrea le preguntan por su futuro en la docencia no transa en su vocación:

Quiero estar haciendo clases en aula toda mi vida, no me veo haciendo otra cosa, a veces lo he pensado, pero no puedo imaginarme en otra cosa, siento que no serviría para otra cosa.

No obstante, reconoce que muchas veces siente miedo de no ser capaz de seguir: 
No quiero perder la esperanza. Yo creo que los profes que la pierden, que ya no pueden más, es porque el sistema los explotó, no son los estudiantes, no, es el sistema que nos va de a poco aplastando y acallando. Eso me da miedo, perder el sentido, la pasión que me trajo hasta aquí, ¿qué voy a hacer en ese momento?

\subsection{LA HISTORIA DE IGNACIO}

Ignacio es el segundo de cuatro hermanos y desde joven tuvo que aprender a vivir de forma más nómade de lo usual, porque su padre, que trabajaba en una empresa que construía carreteras, debía constantemente cambiar de región por trabajo. Estos traslados hicieron que la familia completa aprendiera a adaptarse rápidamente a nuevos contextos, comenzar de nuevo una y otra vez en barrios, grupos de amigos y colegios, en el cual los cuatro hermanos tuvieron diversos tipos de educación y también conocieron distintos contextos y realidades:

Siempre me acuerdo que hacía amigos igual. Yo creo que aprendí que me iba y llegaba rápido. Me acuerdo que no me demoraba una semana y ya teníamos amigos y me gustaba mucho lo de vivir en la naturaleza.

La vida de la familia se desarrolló en su mayoría en el sur del país, donde lo que caracterizaba a Ignacio era su constante pasión por el dibujo:

Era bueno para dibujar desde siempre, mis amigos me pedían dibujos y cosas así.

Cuando se trasladaron finalmente a Santiago, Ignacio completó su enseñanza media en un colegio particular pagado del sector norte de Santiago, donde conoció a una de sus primeras inspiraciones en el mundo de la pedagogía, su profesora de Lenguaje:

Ella quería mostrarnos cosas distintas del mundo, que nosotros exploráramos, eso nunca lo había visto, de hecho, yo no leía nada y con esa profesora me puse a leer, porque entendí la lectura de otra forma.

Otro de los profesores que marcó cómo es Ignacio frente a su alumnado en la actualidad fue su profesor de Ciencias, del que destaca la confianza en las capacidades de los alumnos, para poder plantear su clase de modo diferente y desafiante:

Me acuerdo que él comenzó la clase hablando de filosofía, sobre la historia del pensamiento científico y eso era lo que yo necesitaba, porque cuando me dieron la oportunidad de pensar y de embarrarla yo mismo, ahí me empezó a gustar más estudiar. Ese profesor tenía ese enfoque y nos hablaba cosas más complicadas porque creía que nosotros podíamos aprenderlas.

Ya al final de la enseñanza media, Ignacio motivado por su padre decide ingresar a estudiar Arte a una prestigiosa universidad. Es en la Facultad de Artes donde conoce a otro de los profesores que fueron claves en su formación y posterior carrera como docente. Su profesor de dibujo, le enseñó la importancia de la pasión a la hora de enseñar y cómo esto permeaba en los alumnos: 
Era uno de los pocos profesores que les gustaba hacer clases y lo decía. Tenía mucho ánimo, inventaba sus propios métodos. Era muy apasionado y romántico para enseñar. Era exageradamente disciplinado, pero le ponía pasión, entonces al final te contagiaba y uno hacía todo igual.

Al finalizar su carrera de Arte, Ignacio sufre un quiebre amoroso que lo impulsa a buscar nuevos rumbos, es así como llega a la pedagogía:

Estaba haciendo mi tesis de Arte y había terminado con una polola. Estaba mal, entonces necesitaba hacer algo y siempre mis compañeros me decían que yo era el que enseñaba las cosas. Y después me puse a estudiar pedagogía y el primer año no me gustó nada, porque me caían mal las personas, los profes, pero ya después el segundo año me gustó más porque ahí teníamos la práctica, entonces con los niños me gustaba, con los adultos no tanto.

Las prácticas le permiten involucrarse en la docencia de forma más experiencial. Esas vivencias son las que más valora Ignacio en su formación como profesor, porque para él, más allá de la teoría, la educación toma un papel importante como catalizador de justicia social:

Con la práctica como que se afirmó en mi cabeza que me gustaba esto, que era bueno para hacer eso, educar y también que era necesario, era como algo que tenía que hacer. Debe ser algo así como un acto de justicia, de justicia social. Uno sabe que en colegios vulnerables hay más necesidades que en otro lado. He tenido esas reflexiones y he llegado al punto en que tengo que hacerlo, siempre ando escapando de hacerme cargo de situaciones de la vida y después me doy cuenta que por algo deben llegar a mí, porque yo soy bueno para resolverlas.

Al finalizar sus estudios de pedagogía, Ignacio no comenzó a trabajar inmediatamente como profesor. Para él, un docente o profesional debe conocer el mundo antes de poder enseñarlo a un alumno y fue lo que él hizo. Decidió irse a viajar y trabajar en distintos oficios durante tres años. Esta experiencia le entregó valores y formó su carácter para su posterior carrera docente:

Viajar me aportó flexibilidad de pensamiento, tolerancia con las personas, empatía. Te enriquece el mundo cultural, intelectual, afectivo, emocional.

A la vuelta de su viaje, el año 2015, Ignacio ingresó al Liceo Técnico en el que trabaja actualmente. En el establecimiento, él se haría cargo del departamento y las clases de Arte para primeros y segundos medios, además de una jefatura. El comienzo de su carrera en el Liceo no estuvo exento de miedos, puesto que, como toda nueva experiencia, debía adaptarse a un contexto que no conocía:

Tenía mucho miedo me acuerdo, no cachaba que iba a hacer, además yo estudié para trabajar en colegio humanista y este Liceo es técnico, entonces era todo muy distinto y no me gustaba, así que la pasaba mal, más que nada con los adultos, con los niños me 
entendía mejor, pero como me estaba adaptando igual era mucho trabajo entender de qué se trataba como este país nuevo, yo no entendía.

Tener una jefatura en su primer año también fue un punto difícil para Ignacio, dado que en su formación como profesor reconoce no haber adquirido herramientas al respecto y tampoco haber recibido el apoyo del colegio para poder ejercer ese rol:

No había tanto apoyo de UTP (Unidad Técnica Pedagógica) u orientación, un apoyo como profesional. Entonces dependía mucho de uno y había un grupo de niños que era más complicado de llevar, entonces me costó como un año.

Otro aspecto que afectó a Ignacio en ese tiempo de adaptación fue la relación con el equipo directivo de ese entonces:

Era gente que creía que tenían ideas buenas y que en realidad creo que no habían leído muchos libros. Leyeron dos y pensaban que sabían. Lo único que entendía era que no creían en la capacidad de los niños, siempre los subestimaban por su clase social, por su origen, por su dificultad en la vida. Pero yo creo que en la vida uno tiene que poder superar las cosas si está con más gente que te ayude, pero así solapando no ayudas porque el problema queda igual, no lo enfrentaban.

No obstante, estas formas de trabajar y las claras diferencias de opinión, él se sobrepuso a ellas para poder avanzar en su misión como docente:

Pensé en irme en ese período, pero me quería quedar como para tomar experiencia y buscar después otras cosas. Ahora siento que no, que estoy cómodo y no tengo problemas. Yo tengo la filosofía de que en la vida uno tiene que hacer hartas cosas y seguir un camino que a veces te lleva por corrientes tormentosas, pero otras veces el mar está tranquilo.

Y sin duda, la relación con sus alumnos se configuró como un eje central para permanecer a pesar de todos los problemas:

Me quedé por los chiquillos. Son jóvenes muy abandonados, entonces la única figura que pueden tener de algo decente en la vida es uno. Entonces uno se da cuenta de eso, uno sabe que si se va no hay nada más. Por ejemplo, hay un niño, él siempre llega a la casa y habla de mí, el papá me cuenta, pero tiene muchos problemas en la casa, él vive en un ambiente muy tóxico todo el tiempo y es un buen niño. Yo sé que si no voy un día a clases a él le afecta y si yo me fuera a él también le afectaría.

El año 2017 el equipo directivo completo del establecimiento es removido y asume un nuevo director que calza más con la forma de pensar de Ignacio. Esto él lo califica como el inicio de la calma, porque con esta nueva plana ejecutiva establece una relación transparente, cercana y de confianza, que le permite trabajar con tranquilidad y poder hacer cosas nuevas para sus alumnos: 
La relación ahora es buena, transparente, nos podemos decir las cosas, nos podemos enojar, nos podemos reír, tenemos confianza. A veces no las mismas ideas, pero sí la confianza para decirnos las cosas, no hay miedo.

Otra de las razones para la permanencia de Ignacio en el establecimiento es su propia personalidad, porque de acuerdo con sus propias creencias, hay situaciones que debe afrontar antes de abandonar:

Me gusta enfrentar las cosas, a veces uno tiene que irse de un lugar o de una situación, pero en general me gusta enfrentarlas y resolverlas. Yo creo que eso lo tomé de mi familia, porque mi papá es súper aprensivo, te cuida mucho como si uno fuera un niño. Entonces yo en un momento dije, 'si dejo que mi papá siga haciendo eso, no voy a poder ser yo mismo’, entonces me desafío para romper eso.

Con el tiempo, la relación con el equipo de profesores y UTP también mejora sustantivamente, por lo que Ignacio también se abre al trabajo colaborativo e interdisciplinario, que destaca entre las cualidades del cuerpo docente del Liceo:

La confianza en mi trabajo se refleja en que algunos colegas verbalizan la confianza que tienen ellos en como yo hago las cosas y me lo dicen a mí en privado y en público. Entonces, como que se ahorra en el dialogo 'Ignacio tu hazlo' y no me explican mucho, saben lo que yo voy a hacer y me dejan. $\mathrm{O}$ a veces si tengo que pelear, les encaro lo mismo, el historial, ‘¿cuándo yo he fallado?', ‘ya hazlo’, me dicen.

Con el paso del tiempo, Ignacio también asumió un rol importante en la formación valórica de sus alumnos, a través del arte y también a través de su rol como profesor jefe:

Yo creo que el amor propio, el autocuidado son las cosas que el arte te da, como que te despierta preguntas contigo mismo, conectarte contigo mismo, porque cuando uno crea arte en realidad está hablando de uno y a veces eso es difícil decirlo con otro lenguaje, el arte es lenguaje visual y para algunos muchachos es más fácil, para otros más cómodo porque no está evidentemente nombrado el problema. Por ejemplo, tenían que hacer un autorretrato. Y aparecen cosas súper duras de violencia intrafamiliar, abandonos de madre, que te cuentan ellos en sus historias, si lo han superado o si todavía viven con eso, pero en general todos son muy resilientes, saben sobrellevar las cosas duras de la vida y no olvidarlas, pero vivir con eso aceptando que los hace más fuertes.

Por otro lado, Ignacio reconoce en sí mismo un papel muy definido dentro del establecimiento, por lo que se empodera de ese rol y avanza desde ese lugar en sus relaciones con alumnos, profesores, directivos y apoderados:

Mi rol en el Liceo es como multifacético, es como una membrana que une ciertos canales para que todo funcione bien. ¿Otra metáfora? En el fútbol es el medio campo, el jugador que arma los pases, que va hacia atrás, que arma el juego. Pero no es el capitán siempre. Así uno puede ver todo lo que pasa, aprende también de todos los 
demás, se ve lo que se hace, cuando aciertan y cuando fallan y también que te conozcan a ti, también eso es importante, que te conozcan a ti, tu trabajo y quién eres tú.

Es en ese rol donde, pese a todo, Ignacio ve el sentido de su trabajo, como un acto de justicia social que "debe hacerse" y que por "alguna razón" ha llegado a su vida como desafío:

Debe ser algo así como un sentido de justicia, sabes. Sé que aquí hay más necesidades que en otro lado.

Este sentimiento acompaña todas sus tareas diarias y lo ayuda a entender, que más allá de la exigencia de su posición, ese es el trabajo que le gusta hacer. En el establecimiento también entienden la multiplicidad de roles que tiene un profesor, por lo que han establecido pautas y formas de trato que dejan a todos tranquilos con la labor realizada. Las exigencias se establecen en base a la contingencia y los planes se van modificando, dependiendo de lo que suceda con los alumnos, esa transparencia y claridad en el actuar de sus jefes, sin duda es un punto clave para la permanencia de Ignacio en el liceo en el que trabaja:

Hay que ser como un superhéroe aquí. Porque hay que hacer todo, hay que entrevistar a los papás, hay que hablar con la psicóloga, hay que medirlos, pesarlos, todo eso hay que hacerlo. Hay que llevarlos, conversar con ellos, hay que ir a reuniones de profes, hay que planificar, hay que revisar trabajos. Yo termino haciendo eso acá y más, entonces no me urjo cuando me dicen que me atraso con las planificaciones porque no me da el tiempo, eso les digo y me entienden.

A pesar de estar planificando la realización de un diplomado y magíster, Ignacio admite que lo que más le apasionaría sería continuar su carrera en alguna ciudad más pequeña donde pueda impactar a toda la comunidad:

Es como un ideal, un pueblo donde uno puede como experimentar todo desde cero, sin mucha influencia desde otros lados, trabajar con la comunidad, no es mi idea llegar y decir esto es lo que hay que hacer, no, trabajar con toda la comunidad y buscar una forma de mejorar la comunidad, un impacto mayor, no solo educativamente, más grande. Desde el arte y la educación, claro.

Por otra parte, Ignacio no se ve fuera del aula o el arte, por lo que descarta los cargos directivos de cualquier tipo:

Ayer estaba hablando de eso con unos profes, que algunos querían hacer otras cosas, trabajar en orientación o UTP, yo dije que a mí me gustan las clases, mucho. Yo soy grabador, siempre me ha gustado la idea de tener un taller de grabado con los chiquillos y hacer cosas para que ellos produzcan lo que necesiten, si quieren poleras, banderas, eso sería bacán, pero está difícil.

Es por esta razón que Ignacio espera continuar en el aula, demostrando la importancia que tiene la educación y los educadores en la sociedad y su misión se mantiene intacta, 
seguir enseñando y aprendiendo a la vez, para construir un mejor tejido social y aportar a la vida de más estudiantes:

En la clase yo quiero mostrarles mi mundo a los alumnos, con pasión para hablar de lo que me gusta, porque yo no puedo enseñar algo que no me guste. Tampoco subestimando a las personas. 'Ay, no es que los niños no alcanzan a aprender así que hasta aquí no más'. No, yo les tiro todo a la parrilla.

\section{DISCUSIÓN Y CONCLUSIONES}

Una primera dimensión para destacar es la influencia de las historias de vida y experiencias pasadas de los docentes. Como señalan Priestley et al. (2015) la práctica docente se ve influenciada por las experiencias pasadas de los profesores, así como por las culturas y estructuras de los contextos profesionales iniciales. En los casos estudiados la dimensión iterativa actúa como un elemento que potencia la permanencia. Los docentes refieren a historias familiares que modelaron su carácter y que marcaron de manera importante sus modos de ser en la actualidad. En el caso de Andrea, relata ejemplos de perseverancia, esfuerzo y de pasión, elementos que actualmente despliega en la escuela. Ignacio, refiere una infancia y juventud nómade, que le otorgaron flexibilidad, empatía, tolerancia y mejores posibilidades de adaptación a contextos diversos. Esto es consistente con lo propuesto por Barnatt et al. (2017) quienes mencionan la importancia para una permanencia exitosa el ajuste de la identidad de los docentes durante su despliegue profesional con los desequilibrios y consecuentes acomodamiento con sus entornos laborales.

Adicionalmente, Roness (2011) menciona la relevancia para el posterior ejercicio profesional de las experiencias práctica durante la formación docente. En particular, Freedman y Appleman (2009) señalan que una formación integral en términos prácticos y teóricos favorecen la reflexión docente y esto impacta de manera positiva la permanencia de los profesores en contextos más vulnerables. En este sentido, es interesante que Andrea relata que la vivencia de la práctica reafirma en ella la convicción que desea trabajar en contextos donde "más la necesiten" y esto la impulsa a buscar su primer trabajo en instituciones que atienden contextos que ella cataloga como difíciles. En el caso de Ignacio, la práctica fue una instancia que reafirmó en él el gusto por la docencia, consolidó su confianza en que podía desarrollarlo de buena manera y le entregó sentido a su labor, también menciona que fue esta instancia la que lo incentivó a involucrarse como docente en contextos más vulnerables. En el caso de ambos, las experiencias de prácticas se mencionan como momentos claves en su formación profesional.

Por otra parte, al profundizar en la dimensión evaluativa práctica, es relevante aludir al inicio en la docencia, momento clave en la trayectoria profesional (Hobson et al., 2007). Andrea relata que fue difícil la adaptación, ajustarse a un contexto complejo y cumplir con todas las exigencias y responsabilidades que había adquirido. Durante este periodo destaca el apoyo que recibió del equipo de gestión y de sus pares, que la ayudaron a sobreponerse. En este ámbito, variadas investigaciones en el área puntualizan que el apoyo de los equipos directivos y la colaboración es clave en la permanencia de los docentes (Aspfors y Bondas, 2013; Stockard y Lehman, 2004). Asimismo, es muy relevante el apoyo en la inserción en entornos más desafiantes, el cual permite reducir la presión y agotamiento de los profesores 
(Kelly et al., 2015). Es interesante observar que, al sobreponerse a la iniciación en la escuela, para Andrea es este mismo contexto complejo el que hoy en día se transforma en una fuente de continuidad. La docente reconoce las dificultades de sus alumnos y en esto encuentra un sentido a su labor, tener la oportunidad de aportar en la formación y vida de esos estudiantes es una fuente de persistencia para ella.

En el caso de Ignacio, el inicio en la docencia implica miedo e incertidumbre. Además, no recibe apoyo suficiente del equipo de gestión y de sus pares. Esto genera una primera discontinuidad en su decisión de permanecer. Piensa en irse del colegio, porque además de los desafíos propios del inicio en la profesión, no logra buenas relaciones con el equipo directivo. Cuando se profundiza en cómo logra sobreponerse a esta crisis, hay dos aspectos interesantes de mencionar. El primero, es que emergen características propias de su historia de vida, y en ese momento de cambio tiene la capacidad y flexibilidad de adaptarse, entendiendo que, al ser una nueva experiencia, debe sobreponerse, resolverla y aprender algo nuevo. El segundo elemento interesante es, que al igual que Andrea, encuentra sentido a su labor en ese contexto de adversidad. Ignacio menciona que se quedó por sus estudiantes, porque él es una figura relevante en la vida de esos jóvenes, siendo esto una fuente de persistencia. En este ámbito, resuena en las palabras de ambos docentes un sentido de "deber", de justicia, que ellos la hacen suya a través de su despliegue y compromiso profesional. Parece prudente conjeturar que en sus escuelas se ha logrado una visión compartida por la comunidad escolar, en la cual se declaran altas expectativas para los estudiantes, elemento que autores como Cochran-Smith et al. (2012) han asociado a una mayor permanencia.

Ambos profesores manifiestan un compromiso profundo con los contextos en los cuales se desempeñan y, en particular, expresan un interés por ser agentes transformadores de la realidad en la cual están insertos. Sin embargo, es importante puntualizar que se pone de manifiesto que, aunque las capacidades personales de los profesores son necesarias para la gestión docente, estas no son suficientes (Priestley et al., 2015). En este ámbito, el apoyo y colaboración de los equipos de gestión surgen como experiencias que pueden obstaculizar o favorecer el trabajo docente (Aspfors et al., 2013; Farrell, 2016). Esto es explícito en el caso de Andrea, quien en su inserción reconoce el apoyo y sostén del equipo de gestión y como contrapunto el momento de inflexión en su trayectoria en la escuela, también es provocado por diferencias importantes con los directivos. El relato de Andrea en relación con este episodio marca una discontinuidad en su decisión de permanecer en la institución y solo se soluciona por la desvinculación del equipo directivo. En el caso de Andrea e Ignacio, contar con un equipo de gestión que logra generar un ambiente colaborativo, de confianza y que les otorga autonomía, son narrados como elementos que favorecen la continuidad en las escuelas.

Otro elemento destacable son las relaciones y roles que los docentes han establecido. Por un lado, investigación en esta área menciona que relaciones satisfactorias con los estudiantes favorecen la retención de los docentes (Crocco y Costigan, 2007). Andrea, reconoce que la relación con sus estudiantes es un elemento que la mantiene en la escuela. Cabe mencionar, que la profesora señala sentirse identificada con la comunidad educativa, en particular, ella reconoce en las historias y dificultades de sus estudiantes vivencias propias de su niñez y adolescencia, este reconocimiento opera como una experiencia que fortalece la continuidad en la escuela. Por otra parte, Ignacio relata que sus roles en la escuela son múltiples, y aunque reconoce que esto le acomoda desde el punto de vista que siente han depositado confianza en él, también declara que estos múltiples roles a veces 
lo sobrepasan. En este aspecto, se evidencian dos elementos destacables que lo ayudan a sobreponerse. El primero, es la claridad y transparencia en el actuar de sus jefes que lo ayudan a jerarquizar que es prioritario y que puede esperar. El segundo, es que estos distintos roles surgen como un elemento que parece calzar con la personalidad inquieta de Ignacio, él se describe como una persona que busca hacerse cargo de las situaciones, solucionar los problemas que se le van presentando y superar los desafíos.

En relación con la dimensión proyectiva, es muy interesante el hecho que ambos docentes se conciben como maestros en el largo plazo. Andrea es enfática al señalar que quisiera ser docente toda la vida e Ignacio narra sueños en la docencia que generen un impacto a la comunidad. En este sentido, es posible conjeturar que estas proyecciones deben actuar como elementos que fortalecen su permanencia en la profesión. Probablemente, los contextos de despliegue actuales aún muestran múltiples desafíos por los cuales luchar y comprometerse que mantienen a Andrea e Ignacio involucrados con esas comunidades y en especial con esos estudiantes.

Este estudio tuvo como propósito indagar en las continuidades y discontinuidades de las narrativas de permanencia de profesores principiantes en escuelas con alto índice de vulnerabilidad. Fue posible reconocer en los relatos de los participantes un continuo de decisiones que los llevan a permanecer en las escuelas donde se desempeñan. Un elemento para destacar es que la permanencia de estos docentes principiantes responde a un complejo conjunto de experiencias que los han motivado a buscar, ingresar y persistir en lugares más desaventajados. Sin embargo, a partir de las historias de Andrea e Ignacio también es posible reconocer discontinuidades en dichas decisiones, experiencias que los han llevado a cuestionarse el estar trabajando en sus escuelas y que han dificultado su labor profesional. A partir de sus relatos, ha sido posible identificar una serie de vivencias que iluminan la comprensión del fenómeno en estudio, poniendo de relieve que reducir la comprensión de la permanencia a un único factor o un conjunto de ellos conlleva el riesgo de estrechar fuertemente el entendimiento del fenómeno.

Finalmente, es importante señalar que este trabajo tiene como principal limitación el aproximarse a la experiencia de permanencia de dos docentes en un contexto particular no generalizable. Las biografías corresponden a una composición colaborativa en la cual se recogen historias personales que dependen de los recuerdos y relatos que los docentes decidieron compartir con la investigadora.

\section{REFERENCIAS BIBLIOGRÁFICAS}

Aspfors, J. y Bondas, T. (2013). Caring about caring: newly qualified teachers' experiences of their relationships within the school community. Teachers and Teaching: Theory and Practice 19(3), 243-259. doi: 10.1080/13540602.2012.754158.

Ávalos, B. y Valenzuela, J. P. (2016). Education for all and attrition/retention of new teachers: a trajectory study in Chile. International Journal of Educational Development 49, 279-290. doi: 10.1016/j.ijedudev.2016.03.012

Barber, M. y Mourshed, M. (2008). Cómo hicieron los sistemas educativos con mejor desempeño del mundo para alcanzar sus objetivos. PREAL $\mathrm{N}^{\circ} 41$.

Barnatt, J., Gahlsdorf Terrell, D., D’Souza, L., Jong, C., Cochran-Smith, M., Viesca, K., Gleeson, A., McQuillan, P. y Shakman, K. (2017). Interpreting early career trajectories. Educational Policy, 31(7), 992-1032. doi: 10.1177/0895904815625286. 
Bettini, E., Jones, N., Brownell, M., Conroy, M., Park, Y., Leite, W., Crockett, J. y Benedict, A. (2017). Workload manageability among novice special and general educators: relationships with emotional exhaustion and career intentions. Remedial and Special Education, 38(4), 246-256. doi: $10.1177 / 0741932517708327$.

Biesta, G. y Tedder, M. (2007). Agency and learning in the lifecourse: Towards an ecological perspective. Studies in the Education of Adults, 39(2), 132-149. doi: 10.1080/02660830.2007.11661545.

Boyd, D. Lankford, H., Loeb, S. y Wyckoff, J. (2005). Explaining the short careers of high-achieving teachers in schools with low-performing students. American Economic Review, 95(2), 166-171. doi: $10.1257 / 000282805774669628$

Borman, G. D. y Dowling, N. M. (2008). Teacher attrition and retention: a meta-analytic and narrative review of the research. Review of Educational Research, 78(3), 367-409. doi: $10.3102 / 0034654308321455$.

Carrasco, D., Godoy, M. I. y Rivera, M. (2017). Rotación de profesores en Chile: quiénes son y cuál es el contexto de quiénes dejan su primer trabajo. Midevidencias, 11, 1-7.

Carrasco, D., Manzi, J. y Treviño, E. (2018). Trayectorias laborales de los docentes: ¿dónde, cuándo y bajo qué condiciones dejan su primer trabajo? Temas de la Agenda Pública, 13(105), 1-20.

Cochran-Smith, M., McQuillan, P., Mitchell, K., Terrell, D. G., Barnatt, J., D’ Souza, L., Jong, C., Shakman, K., Lam, K. y Gleeson, A. M. (2012). A longitudinal study of teaching practice and early career decisions: a cautionary tale. American Educational Research Journal, 49(5), 844-880. doi: 10.3102/0002831211431006

Clandinin, D. J., Schaefer, L. y Downey, C. A. (2014). Narrative conceptions of knowledge: towards understanding teacher attrition. United Kingdom: Emerald Publishing Ltd.

Cornejo, M., Mendoza, F. y Rojas, R. (2008). La investigación con relatos de vida: pistas y opciones del diseño metodológico. Psykhe, 17(1), 29-39. doi: 10.4067/S0718-22282008000100004

CPEIP (2017). Orientaciones Sistema de Desarrollo Profesional Docente. Santiago: MINEDUC.

Crocco, M. S. y Costigan, A. T. (2007). The narrowing of curriculum and pedagogy urban educators speak out. Urban Education, 42(6), 512-535. doi: 10.1177/004208590730496

Denzin, N. K. (1989). Interpretive Biography. Estados Unidos: Sage.

Day, C. (2008). Committed for life? Variations in teachers' work, lives and effectiveness. Journal of Educational Change, 9(3), 243-260. doi: 10.1007/s10833-007-9054-6

Dupriez, V., Delvaux, B. y Lothaire, S. (2016). Teacher shortages and attrition: why do they leave? British Educational Research Journal, 42(1), 21-39. doi: 10.1002/berj.3193

Farrell, T. S. C. (2016). Surviving the transition shock in the first year of teaching through reflective practice. System, 61, 12-19. doi: 10.1016/j.system.2016.07.005

Feng, L. (2010). Hire today, gone tomorrow: new teacher classroom assignments and teacher mobility. Education Finance and Policy, 5(3), 278-316. doi: 10.1162/EDFP_a_00002

Freedman, S.W. y Appleman, D. (2009). "In it for the Long Haul": how teacher education can contribute to teacher retention in high-poverty, urban schools. Journal of Teacher Education, 60(3), 323-337. doi: 10.1177/0022487109336181

Gaikhorst, L., Beishuizen, J., Roosenboom, B. y Volman, M. (2017). The challenges of beginning teachers in urban primary schools. European Journal of Teacher Education, 40(1), 46-61. doi: $10.1080 / 02619768.2016 .1251900$

Harfitt, G. J. (2015). From attrition to retention: a narrative inquiry of why beginning teachers leave and then rejoin the profession. Asia-Pacific Journal of Teacher Education, 43(1), 22-35. doi: 10.1080/1359866X.2014.932333

Harper, D. (2002). Talking about pictures: a case for photo elicitation. Visual Studies, 17(1), 13-26.

Heikonen, L., Pietarinen, J., Pyhältö, K., Toom, A. y Soini, T. (2016). Early career teachers' sense of professional agency in the classroom: associations with turnover intentions and perceived inadequacy in teacher-student interaction. Asia-Pacific Journal of Teacher Education, 40(3), 250-266. doi: 10.1080/1359866X.2016.1169505 
Hernández, R., Fernández, C. y Baptista, P. (2014). Metodología de la Investigación (6th ed.). México: McGraw Hill Education.

Hobson, A. J., Malderez, A., Tracey, L., Homer, M., Mitchell, N., Biddulph, M., Giannakaki, M. S., Rose, A., Pell, R. G., Roper, T., Chambers, G.N. y Tomlinson, P. D. (2007). Newly qualified teachers' experience on their first year of teaching. Findings from phase III of the becoming a teacher project. Nottingham: University of Nottingham, University of Leeds and Ipsos MORI Social Research Institute.

Kelly, S. y Northrop, L. (2015). Early career outcomes for the "best and the brightest": selectivity, satisfaction, and attrition in the beginning teacher longitudinal survey. American Educational Research Journal, 52(4), 624-656. doi: 10.3102/0002831215587352

Lavigne, A. L. (2014). Beginning teachers who stay: beliefs about students. Teaching and Teacher Education, 39, 31-43. doi: 10.1016/j.tate.2013.12.002

Mishler, E. G. (1986). Research interviewing: Context and narrative. Cambridge, MA: Harvard University Press.

Mourshed, M., Chijioke, C. y Barber, M. (2010). How the world's most improved school systems keep getting better. New York: McKinsey \& Company.

OCDE (2005). Teachers matters: Attracting, developing and retaining effective teachers. Paris: OCDE Publishing.

Priestley M., Biesta G. y Robinson S. (2015) Teacher Agency: An Ecological Approach. London: Bloomsbury Academic.

Riessman, C. K. (2008). Narrative methods for the human sciences. California: Sage Publications.

Roness, D. (2011). Still motivated? The motivation for teaching during the second year in the profession. Teaching and Teacher Education, 27(3), 628-638. doi: 10.1016/j.tate.2010.10.016

Schaefer, L., Long, J. S. y Jean Clandinin, D. (2012). Questioning the research on early career teacher attrition and retention. Alberta Journal of Educational Research, 58(1), 106-121.

Stockard, J. y Lehman, M. B. (2004). Influences on the satisfaction and retention of 1st-year teachers: the importance of effective school management. Educational Administration Quarterly, 40(5), 742-771. doi: 10.1177/0013161X04268844.

Valenzuela, J. P. y Sevilla, A. (2013). La movilidad de los nuevos profesores chilenos en la década del 2000: un sistema escolar viviendo en peligro, Fondecyt $N^{\circ} 1120740$, Santiago: Centro de Investigación Avanzada en Educación (CIAE), Universidad de Chile. 